\title{
MauG catalysis: Chemistry from a distance.
}

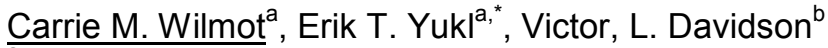

${ }^{a}$ Dept. of Biochem., Molecular Biology \& Biophysics, University of Minnesota, St Paul, MN 55108, USA

${ }^{\mathrm{b}}$ Burnett School of Biomedical Sciences, University of Central Florida, Orlando, FL 32827, USA

*Current address : Dept. of Chem. \& Biochem., New Mexico State University, Las Cruces, NM 88003, USA

Methylamine dehydrogenase (MADH) is a periplasmic enzyme found in a range of methylotrophic / autotrophic bacteria that enables the organisms to grow on methylamine as a sole source of carbon and energy. Catalytic activity requires the posttranslational modification of two Trp residues in MADH to form the 2-electron redox cofactor tryptophan tryptophylquinone (TTQ), in which two carbonyl oxygens have been added to one of the Trp indole rings, and the two Trp residues crosslinked. Maturation of MADH requires the expression of four accessory genes. One of these is a di-heme enzyme, MauG, which catalyzes the completion of TTQ biosynthesis in a 6-electron oxidation. The key MauG catalytic intermediate is a bis- $\mathrm{Fe}(\mathrm{IV})$ species comprised of a ferryl heme $(\mathrm{Fe}(\mathrm{IV})=\mathrm{O})$ with the second heme in the $\mathrm{Fe}(\mathrm{IV})$ oxidation state. Completion of TTQ to generate active MADH involves long-range electron transfer and a radical hopping mechanism to effect catalysis over a $40 \AA$ distance. The MauG catalyzed reaction occurs in three discrete 2-electron events in a $\mathrm{H}_{2} \mathrm{O}_{2}$ or $\mathrm{O}_{2}$-dependent process. A crystal structure of MauG in complex with its protein substrate, a precursor form of MADH known as preMADH, has been solved. The crystals are catalytically active. The order of the 2-electron chemistry catalyzed by MauG was determined in the crystal and corroborated by mass spectrometry in solution. This talk will give background on studying enzyme reactions within crystals, and detail how we combined crystallography and mass spectrometry to determine the order of chemistry in TTQ biosynthesis. 